University of Wollongong

Research Online

Faculty of Engineering and Information

Faculty of Engineering and Information

Sciences - Papers: Part B

Sciences

2018

An image recognition method for gear fault diagnosis in the manufacturing line of short filament fibres

Shoufeng Jin

Xi'An Polytechnic University

Di Fan

Xi'An Polytechnic University

Reza Malekian

University of Pretoria

Zhihe Duan

Xi'an Jiaotong University

Zhixiong $\mathrm{Li}$

University of Wollongong, zhixiong.li@ieee.org

Follow this and additional works at: https://ro.uow.edu.au/eispapers1

Part of the Engineering Commons, and the Science and Technology Studies Commons

Research Online is the open access institutional repository for the University of Wollongong. For further information contact the UOW Library: research-pubs@uow.edu.au 


\title{
An image recognition method for gear fault diagnosis in the manufacturing line of short filament fibres
}

\author{
Abstract \\ The manufacturing line is a fundamental element in short filament fibre production, in which the gearbox \\ is the key mechanical part. Any faults in the gearbox will greatly affect the quality of the short filament \\ fibres. However, due to the harsh working environment, the gearbox is vulnerable to failure. Due to the \\ complexity of the manufacturing line, effective and efficient feature extraction of gear faults is still a \\ challenge. To this end, a new fault diagnosis method based on image recognition is proposed in this \\ paper for gear fault detection in fibre manufacturing lines. In this method, wavelet packet bispectrum \\ analysis (WPBA) is proposed to process the gear vibration signals. The bispectrum texture is obtained \\ and then analysed by an image fusion algorithm for texture feature extraction. The grey-level co- \\ occurrence matrix is used in the image fusion and the extracted texture features are four parameters of \\ the grey-level co-occurrence matrix. Finally, a support vector machine (SVM) is adapted to recognise the \\ gear fault type and location. Experimental data acquired from a real-world manufacturing line of short \\ filament fibres are used to evaluate the performance of the proposed image-based gear fault detection \\ method. The analysis results demonstrate that the newly proposed method is capable of accurate gear \\ fault det ection in fibre manufacturing lines.

\section{Disciplines} \\ Engineering | Science and Technology Studies

\section{Publication Details} \\ Jin, S., Fan, D., Malekian, R., Duan, Z. \& Li, Z. (2018). An image recognition method for gear fault diagnosis \\ in the manufacturing line of short filament fibres. Insight: Non-Destructive Testing and Condition \\ Monitoring, 60 (5), 270-275.
}




\title{
An image recognition method for gear fault diagnosis in the manufacturing line of short filament fibres
}

\author{
Shoufeng Jin, Di Fan, Reza Malekian, Zhihe Duan and Zhixiong Li
}

\begin{abstract}
The manufacturing line is a fundamental element in short filament fibre production, in which the gearbox is the key mechanical part. Any faults in the gearbox will greatly affect the quality of the short filament fibres. However, due to the harsh working environment, the gearbox is vulnerable to failure. Due to the complexity of the manufacturing line, effective and efficient feature extraction of gear faults is still a challenge. To this end, a new fault diagnosis method based on image recognition is proposed in this paper for gear fault detection in fibre manufacturing lines. In this method, wavelet packet bispectrum analysis (WPBA) is proposed to process the gear vibration signals. The bispectrum texture is obtained and then analysed by an image fusion algorithm for texture feature extraction. The grey-level co-occurrence matrix is used in the image fusion and the extracted texture features are four parameters of the grey-level co-occurrence matrix. Finally, a support vector machine (SVM) is adapted to recognise the gear fault type and location. Experimental data acquired from a real-world manufacturing line of short filament fibres are used to evaluate the performance of the proposed image-based gear fault detection method. The analysis results demonstrate that the newly proposed method is capable of accurate gear fault detection in fibre manufacturing lines.
\end{abstract}

Keywords: filament fibre, manufacturing line, gear fault diagnosis, wavelet packet bispectrum analysis, image fusion.

\section{Introduction}

The manufacturing line for short filament fibres is one of the most important elements in the textile industry. With the improvements in polyester staple fibre technology in recent years, the requirements of short wire production have increased to a high level. The filament is the essential element in forming short wires and the quality of filament production is mainly controlled by the stable and smooth operation of the gearbox transmission system. Unexpected failures on any gears will decrease the quality of the filament ${ }^{[1-3]}$ and eventually reduce the overall performance of the fibre manufacturing line, leading to significant economic losses.

Until recently, time domain analysis ${ }^{[4,5]}$, frequency domain analysis ${ }^{[6]}$ and time-frequency domain analysis ${ }^{[7,8]}$ have been applied to the fault diagnosis of gearboxes. Li et al ${ }^{[9]}$ proposed a multichannel-based vibration analysis method for gear crack detection. Wang and Albert ${ }^{[10]}$ developed an autoregressive model-based filter to detect gear faults and the experimental analysis results suggest a high kurtosis of the filtered signal using the autoregressive model. Ibrahim and Albarbar ${ }^{[11]}$ used Wigner-Ville distribution to analyse the empirical mode decomposition of helical gear vibration to diagnose gear failures. Jena et al ${ }^{[12]}$ adopted active noise cancellation to reduce the background noise in the gear vibration and then used a wavelet transform to extract gear fault features. Hreha et al ${ }^{[13]}$ put forward a fault diagnosis model based on wavelet denoising and principal component analysis (PCA) for detecting multiple gear faults. Many further references have discussed the application of vibration analysis to the condition monitoring and fault diagnosis (CMFD) of gearboxes ${ }^{[14]}$.

A literature review indicates that time-frequency analysis of the gear vibration signal is the most popular and effective method for discovering progressing failures ${ }^{\mid 15]}$. The latest publications ${ }^{[16,17]}$ show the promising performance of image-based fault detection techniques. An image processing technique can address the challenges of fault feature extraction by overcoming the limitations of manual feature selection ${ }^{[16]}$. Since an image processing technique is able to comprehensively analyse the fault characteristics based on high-dimensional image data of the gearbox vibration, better features can be extracted by the image-based method than those of traditional time-frequency analysis. However, to the best knowledge of the authors, image-based fault detection has not been attempted for the fault diagnosis of gearboxes in fibre manufacturing lines.

To address the aforementioned issue, this paper proposes a new method using image-based fault detection to detect gear faults in fibre manufacturing lines. The aim of the present work is to develop a new vibration image extraction method based on wavelet packet bispectrum analysis (WPBA) and the grey-level co-occurrence matrix (GLCM). The gear vibration signal is first transformed into a vibration image using WPBA. Then, the GLCM is applied to the images to extract the fault features. Lastly, the gear fault type

\section{- Submitted 25.09.17/ Accepted 18.12.17}

Shoufeng Jin and Di Fan are with the Department of Mechanical Engineering, Xi'an Polytechnic University, Xi'an 710048, PR China.

Reza Malekian is with the Department of Electrical, Electronic and Computer Engineering, University of Pretoria, Pretoria 0002, South Africa.

Zhihe Duan is with the Department of Mechanical Engineering, Xi'an Jiaotong University, Xi'an 710011, PR China.

Zhixiong Li is with the School of Mechanical, Materials, Mechatronic and Biomedical Engineering, University of Wollongong, Wollongong, New South Wales 2522, Australia and also the School of Mechatronic Engineering, China University of Mining and Technology, Xuzhou 221110, PRChina. 
and location are identified by a support vector machine (SVM). Experimental data has been used to validate the effectiveness of the proposed image-based method.

\section{Image-based gear fault detection method}

\subsection{Gear vibration in fibre manufacturing lines}

Without loss of generality and under conditions of good lubrication and low friction in a gear pair, the frictional force on the tooth surface can be ignored and the gear vibration can be described as:

$$
\frac{m_{1} m_{2}}{m_{1}+m_{2}} \ddot{X}+C \dot{X}+k(t) X=k(t) E_{1}+k(t) E_{2}(t) \ldots
$$

where $m_{1}$ and $m_{2}$ are the masses of the driving wheel and the driven wheel, respectively, $X$ is the relative displacement of the gears along the meshing line, $C$ is the gear meshing damping, $k(t)$ is the gear meshing stiffness, $E_{1}$ is the average static elastic deformation of the gear pair and $E_{2}(t)$ is a failure function.

In Equation (1), the left-hand terms represent the vibration characteristics of the gear pair and the right-hand terms represent the excitation function, in which $k(t)$ is a periodic variable. Generally, $k(t)$ is not a standard sine function, so the harmonic components of the meshing frequency are included. The term $k(t) E_{2}(t)$ in the excitation function is the excitation source of the gear pair, so the task of fault diagnosis is to extract abnormal vibration characteristics caused by fault excitation sources. If a gear fails, the characteristics of the vibration signal can usually be regarded as amplitude modulation and phase modulation, and the mathematical model of the gear vibration signal can be expressed as:

$$
X=\sum_{k=1}^{M} A_{k}\left(1+r_{k}(t)\right) \cos \left(2 \pi k f_{m} t+\varphi_{k}(t)\right)
$$

where $M$ is the harmonic order, $f_{m}$ is the frequency of the carrier signal and $r_{k}(t)$ and $\varphi_{k}(t)$ are the amplitude-modulating and phasemodulating signals, respectively.

\subsection{Wavelet packet bispectrum analysis}

The vibration signal of a gear pair includes not only information about the running state of the gear but also Gaussian and nonGaussian noise. The wavelet packet transform can decompose the vibration signal into high-frequency and low-frequency bands ${ }^{[18]}$. The wavelet packet decomposition is described in Equation (3):

$$
\left\{\begin{array}{l}
\varphi(t)=\sqrt{2} \sum_{l \in Z} h_{l} \phi(2 t-l) \\
\psi(t)=\sqrt{2} \sum_{l \in Z} g_{l} \psi(2 t-l)
\end{array}\right.
$$

where $h$ and $g$ are the high-pass and low-pass wavelet filters, respectively, $\varphi(t)$ is a scaling function and $\psi(t)$ is a wavelet function. In general, the fault information is located in the low-frequency band of the gear vibration signal, so the low-frequency band signal $Y(t)$ can be reconstructed by the wavelet packet transform ${ }^{[18]}$. That is, only the wavelet coefficients that are generated by $\varphi(t)$ and $\psi(t)$ in the specified range of the low-frequency band are preserved, while the wavelet coefficients outside of the specified frequency band are discarded. A time waveform, $Y(t)$, can be reconstructed from the preserved wavelet coefficients. Then, the three-order spectrum can be calculated on $Y(t)$ by Equation (4):

$$
C_{3 x}\left(\tau_{1}, \tau_{2}\right)=E\left[Y(t) Y\left(t+\tau_{1}\right) Y\left(t+\tau_{2}\right)\right]
$$

Hence, the bispectrum of $Y(t)$ can be obtained by a two-dimensional
Fourier transform (FT), as shown in Equation (5):

$$
B_{x}\left(\omega_{1}, \omega_{2}\right)=\sum_{\tau_{1}=-\infty}^{+\infty} \sum_{\tau_{2}=-\infty}^{+\infty} C_{3 x}\left(\tau_{1}, \tau_{2}\right) e^{-j\left(\omega_{1} \tau_{1}+\omega_{2} \tau_{2}\right)}
$$

It can be seen from Equation (5) that the bispectrum is a plural signal ${ }^{[19]}$ describing both the amplitude and phase information of the signal $Y(t)$.

\subsection{Wavelet image fusion}

The bispectrum image can be obtained using WPBA. To extract the texture feature of the image, an image fusion method is employed in this paper based on a wavelet transform and matching degree. Assume two images, $A$ and $B$. Let the wavelet coefficients of these two images, decomposed by a wavelet transform to level $j$, be denoted by $\left\{c A, d A_{j}^{e}\right\}$ and $\left\{c B, d B_{j}^{e}\right\}$, respectively. The wavelet coefficients of the fused image $F$ are $\left\{c F, d F_{j}^{\varepsilon}\right\}$. Here, $c X(X=A, B$ or $F)$ is used to represent the low-frequency coefficients of image $X$ and $d X^{\varepsilon}$ is used to represent the high-frequency wavelet coefficients of image $X$.

For the low-frequency wavelet sub-band $(m, n)$, the wavelet coefficients of the fused image are then the averages of the wavelet coefficients of the original images, as expressed in Equation (6):

$$
c F(m, n)=\frac{1}{2}[c A(m, n)+c B(m, n)] .
$$

where $m$ denotes the decomposition level and $n$ denotes the wavelet note in that level.

Let $E X_{j}^{\varepsilon}$ be the energy of the image in the wavelet sub-bands $(m, n)$ to describe the saliency of the information contained in the high-frequency region of the image:

$$
E X_{j}^{\varepsilon}(m, n)=\sum_{p=-1}^{1} \sum_{q=-1}^{1} R(p+2, q+2)\left[d X_{j}^{\varepsilon}(m+p, n+q)\right]^{2} \ldots
$$

where $R$ is a weight matrix and $p$ and $q$ are wavelet level and note selectors, respectively.

Then, the matching degree of images $A$ and $B$ in the wavelet sub-bands can be expressed as:

$$
\operatorname{MAB}_{j}^{c}(m, n)=\frac{2 \sum_{n=-1}^{1} \sum_{q^{m-1}}^{1} R(p+2, q+2) d A(m+p, n+q) d B_{j}^{c}(m+p, n+q)}{E A_{j}^{\varepsilon}(m, n)+E B_{j}^{c}(m, n)} . .
$$

where $M A B_{j}^{e}$ is the matching degree of images $A$ and $B$. If the matching degree is less than $0.5^{\circ}$, then the wavelet coefficients of the fusion image are calculated using Equation (9):

$$
d F_{j}^{\varepsilon}(m, n)=\left\{\begin{array}{l}
d A_{j}^{\varepsilon}(m, n), \text { if } E A_{j}^{\varepsilon}(m, n) \geq E B_{j}^{\varepsilon}(m, n) \\
d B_{j}^{\varepsilon}(m, n), \text { if } E A_{j}^{\varepsilon}(m, n) \leq E B_{j}^{\varepsilon}(m, n)
\end{array} \ldots . .\right.
$$

If the matching degree is larger than $1^{\circ}$, then the wavelet coefficients of the fusion image are calculated using Equation (10):

$d F_{j}^{\varepsilon}(m, n)=\left\{\begin{array}{l}\omega_{L} d A_{j}^{\varepsilon}(m, n)+\omega_{s} d B_{j}^{\varepsilon}(m, n), \text { if } E A_{j}^{\varepsilon}(m, n) \geq E B_{j}^{\varepsilon}(m, n) \\ \omega_{S} d A_{j}^{\varepsilon}(m, n)+\omega_{L} d B_{j}^{\varepsilon}(m, n), \text { if } E A_{j}^{\varepsilon}(m, n)<E B_{j}^{\varepsilon}(m, n)\end{array} \ldots\right.$

where $\omega_{L}=\frac{1}{2}+\frac{1}{2}\left(\frac{1-M A B_{j}^{\varepsilon}(m, n)}{1-t h r}\right), t h r\left(=0.5^{\circ} \sim 1^{\circ}\right)$ is the matching threshold and $\omega_{S}=1-\omega_{L}$.

The image fusion process is described in Figure 1.

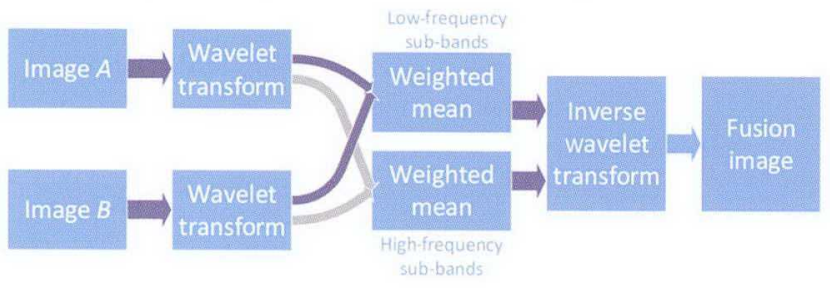

Figure 1. Proposed image fusion process 


\subsection{Feature extraction}

The GLCM is used to extract the image features and represents the probability density of two grey levels in the image in a specified direction ${ }^{[20]}$. Let the positions of the two pixels in the image be $\left(x_{1}, y_{1}\right)$ and $\left(x_{2}, y_{2}\right)$ and the grey values be $i$ and $j$, respectively. Four GLCMs can be obtained for a specified distance, $d$, and for four angles of the image $\left(0^{\circ}, 45^{\circ}, 90^{\circ}\right.$ and $\left.135^{\circ}\right)$. In this way, four features are calculated from the GLCMs:

- Second-order moment, $W_{1}$ :

$$
W_{1}=\sum_{i=1}^{g} \sum_{j=1}^{g} p^{2}(i, j, d, \theta)
$$

where $p$ is the GLCM. The second-order moment is also called energy, which is the sum of the elements of the GLCM. It represents the distribution of the grey level of the image and the size of the texture. The larger $W_{1}$ is, the worse the image texture is.

- Contrast ratio, $W_{2}$ :

$$
W_{2}=\sum_{i=1}^{g} \sum_{j=1}^{g}(i-j)^{2} p^{2}(i, j, d, \theta)
$$

The contrast ratio represents the degree of clarity of the image. The image is clearer when $W_{2}$ is larger.

- Relevance, $W_{3}$ :

$$
W_{3}=\frac{\sum_{\substack{g-1 \\ i=0}}^{\substack{g-1 \\ j=0}}(i \times j \times p(i, j, d, \theta))-u_{x} \times u_{y}}{\sigma_{x} \times \sigma_{y}} \ldots \ldots . .
$$

where $u_{x}$ and $u_{y}$ are the means of the image matrix along the $x$ - and $y$-directions, respectively, and $\sigma_{x}$ and $\sigma_{y}$ are the variances of the image matrix. The relevance represents the texture direction of the image. If the image is strong in a certain direction, $W_{3}$ for that direction will be greater than for the other directions.

- Entropy, $W_{4}$ :

$$
W_{4}=-\sum_{i=1}^{g} \sum_{j=1}^{g} p(i, j, d, \theta) \log _{10} p(i, j, d, \theta)
$$

Entropy represents the complexity of the image. The image texture is more complex when $W_{4}$ is larger.

In order to fully exploit the texture features of the fusion image, the GLCM in the four directions is weighted in the following manner:

$$
S(i, j)=a_{1} p_{0}(i, j)+a_{2} p_{45^{\circ}}(i, j)+a_{3} p_{90^{\circ}}(i, j)+a_{4} p_{135^{\circ}}(i, j) \ldots
$$

where $a_{1}, a_{2}, a_{3}$ and $a_{4}$ are weighting coefficients.

\subsection{Overview of the proposed method}

Figure 2 depicts an overview of the proposed image-based gear fault detection method. The detailed steps in the Figure are described as follows:

Step 1: Collect the vibration signals of the gear pair using accelerometers.

Step 2: Construct the bispectrum images of the vibration signals using WPBA.

Step 3: Fuse the original images and extract the four features using the image fusion and GLCM methods.

Step 4: Diagnose the gear faults using the SVM.

\section{Experimental results and discussion}

Experimental data collected from a real-world fibre manufacturing line have been used to evaluate the proposed image-based gear fault diagnosis method.

\subsection{Experimental data}

The multi-stage fixed-axle gearbox in the short wire manufacturing line is shown in Figure 3. The configuration of the gearbox is shown in Figure 4. In the experimental tests, the gear faults were applied to Gear 1 on the input axle in Figure 4. The gear fault types included a broken tooth, a cracked tooth and a missing tooth, as shown in Figure 5. The driven speed of the input axle was $1600 \mathrm{r} / \mathrm{min}$ and the gear meshing frequency was $f=994 \mathrm{~Hz}$. The vibration signals were recorded by accelerometers in the horizontal and vertical directions of the transmission axle. The sampling frequency was $6840 \mathrm{~Hz}$ and 230 samples of each faulty condition were collected.

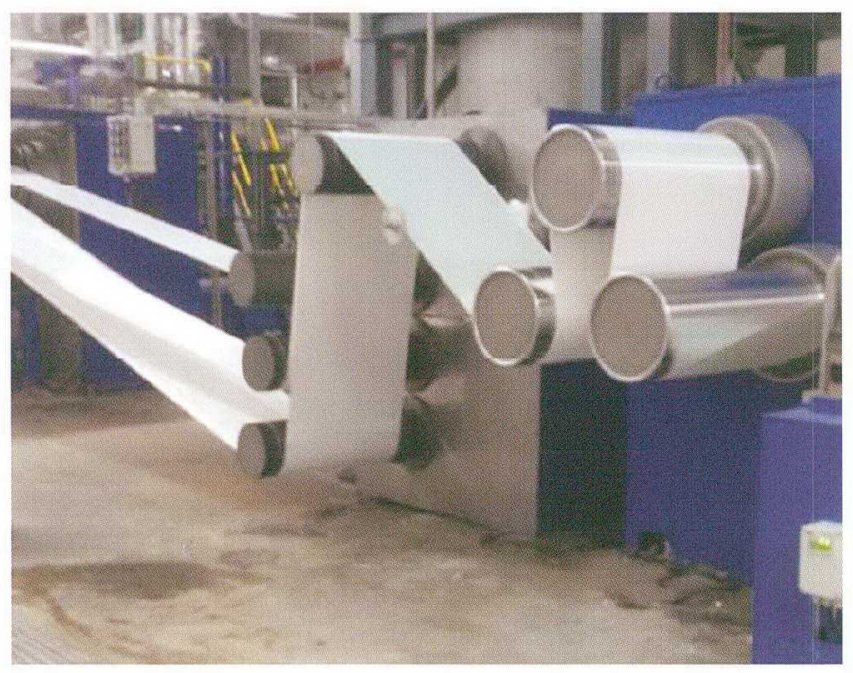

Figure 3. Gearbox experimental platform

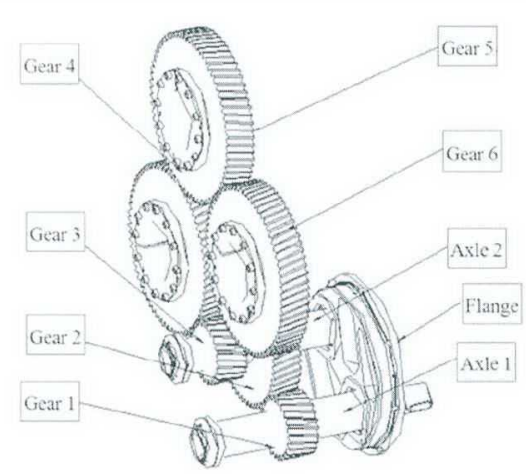

Figure 4. Gearbox configuration 


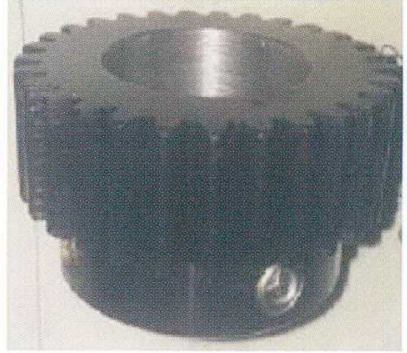

(a)

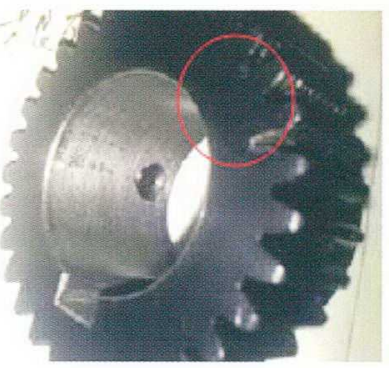

(c)

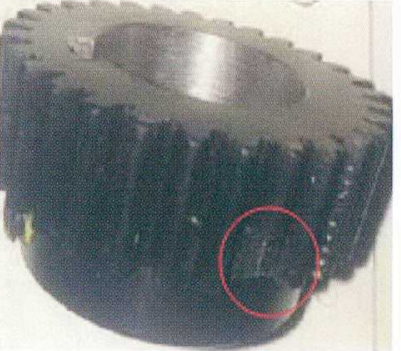

(b)

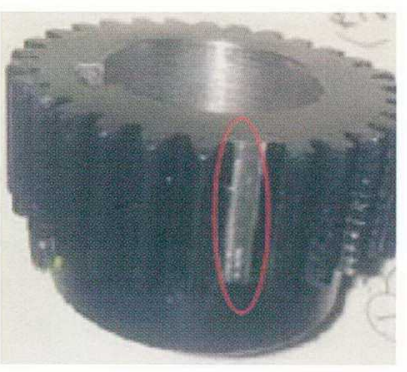

(d)
Figure 5. Gears with different fault types in the experimental tests: (a) normal gear; (b) broken tooth; (c) cracked tooth; and (d) missing tooth

\subsection{Experimental results}

Figure 6 depicts the raw vibration signals of the tested gears under different faults. Their corresponding bispectrum images are provided in Figure 7. As can be seen in the Figure, the bispectrum distributions were significantly different for each operating condition of the gearbox. In Figure 7(a) the normal (non-faulty) gear generated a bispectrum with a high energy concentration at only the first two harmonics ( $f$ and $2 f$ ) of the gear meshing frequency of the input shaft. In contrast, in Figure $7(\mathrm{~b})$ the bispectrum distribution for a broken tooth presented obvious impulsive components around $3 f$. In Figure 7(c), in addition to energy spikes at $f$ and $2 f$ of the gear meshing frequency of the input shaft, there was a large amount of background noise in the bispectrum image. In Figure 7(d), clear sideband energy spikes are seen located around the main energy at $f$ and $2 f$. Although some differences have been found in the bispectrum images of the gear vibration signals under different conditions, it is difficult to distinguish each gear fault directly from Figure 7.

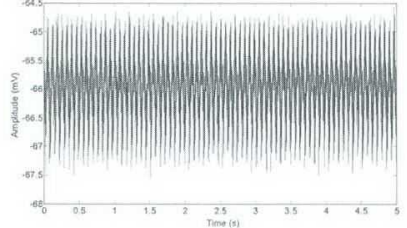

(a)

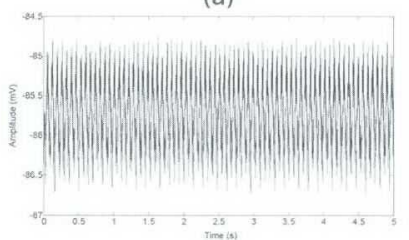

(c)

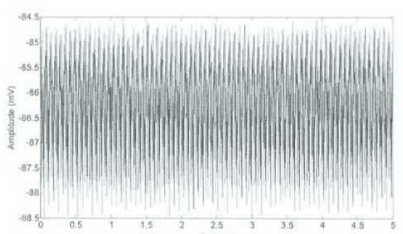

(b)

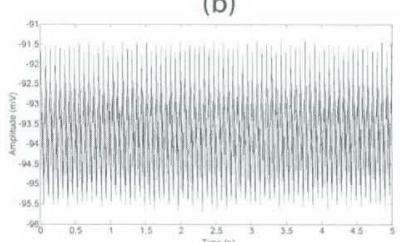

(d)
Figure 6. Raw vibration signals of tested gears under different faults: (a) normal gear; (b) broken tooth; (c) cracked tooth; and (d) missing tooth

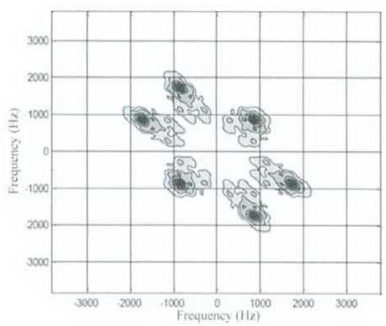

(a)

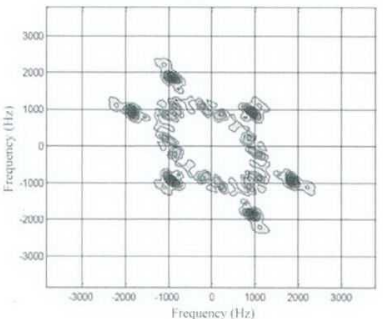

(c)

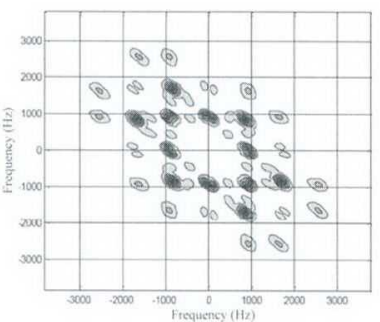

(b)

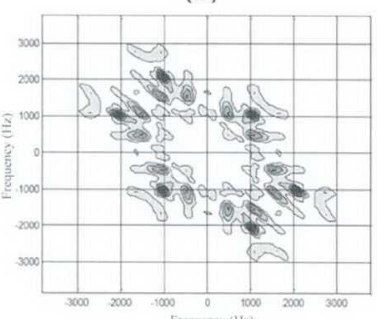

(d)
Figure 7. Original bispectrum images of gear vibrations under different conditions: (a) normal gear; (b) broken tooth; (c) cracked tooth; and (d) missing tooth

The original image was then processed by WPBA and the WPBA-based images are shown in Figure 8. It can be seen that, compared with Figure 7, the noise interference in Figure 8 was greatly reduced after the WPBA processing, the texture of the images was much clearer after the WPBA processing and the main energy spikes of the four gear operating conditions were concentrated at $f$ and $2 f$ of the gear meshing frequency of the input shaft. Due to the symmetry of the higher-order spectrum, the bispectrum distributions were centrosymmetric in Figure 8 under the four gear operating conditions. These observations are very useful for gear fault detection using the image processing technique because of the centrosymmetric bispectrum distributions.

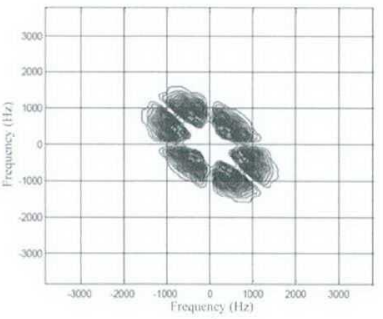

(a)

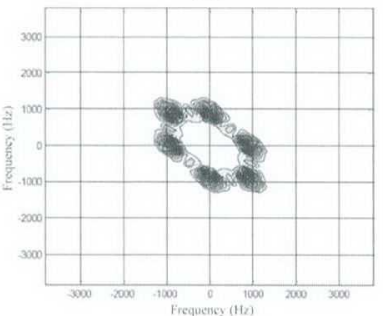

(c)

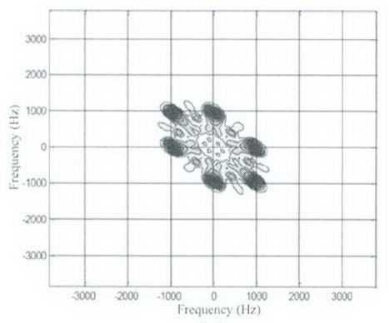

(b)

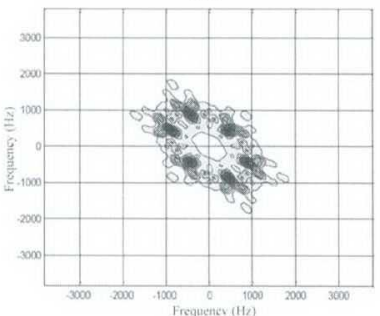

(d)
Figure 8. WPBA-based images of gear vibrations under different conditions: (a) normal gear; (b) broken tooth; (c) cracked tooth; and (d) missing tooth

In order to fully exploit the characteristics of the gear fault signal, the image fusion method proposed in Section 2.3 was applied to the WPBA-based images of the gear vibration signals under the four gear operating conditions. Figure 9 shows the fused image results. 
Comparing Figures 8 and 9, it can be noted that the energy spikes in Figure 9 are concentrated much more precisely at $f$ and $2 f$ of the gear meshing frequency of the input shaft than those in Figure 8. Therefore, the fused bispectrum images present much more reliable fault characteristics than the non-fused ones.

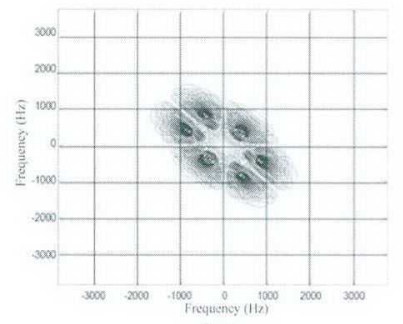

(a)

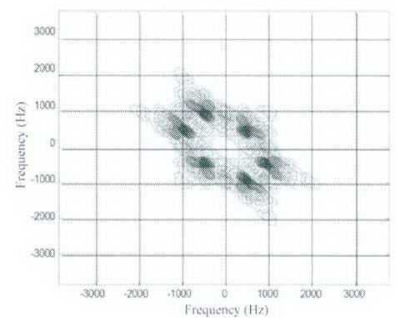

(c)

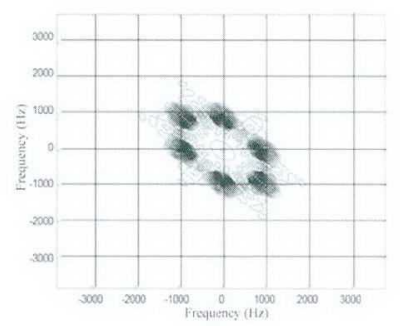

(b)

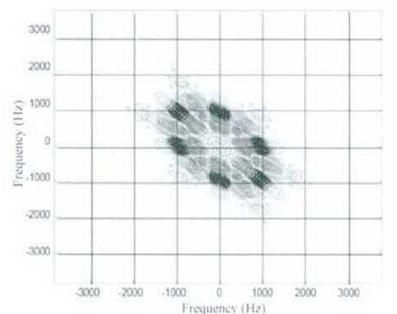

(d)
Figure 9. Fusion images of gear vibrations under different conditions: (a) normal gear; (b) broken tooth; (c) cracked tooth; and (d) missing tooth

The four features of the fused images listed in Section 2.4 $\left(W_{1}-W_{4}\right)$ were extracted using the GLCM. The GLCM features were then input into an SVM classifier for fault identification. In the training process of the SVM model, 130 samples of each operating condition of the gearbox were used as the training datasets and the other 100 samples of each operating condition were treated as the testing datasets. Table 1 lists the fault detection results using the SVM with different structural parameters and Table 2 gives the fault detection results for each gear condition.
In this work, three popular SVM kernel functions were used in the fault recognition ${ }^{[2,22]}$, ie the radial basis, polynomial and sigmoid kernels. The radial basis kernel is expressed as:

$$
K\left(\mathbf{c}_{1}, \mathbf{c}_{2}\right)=\exp \left(-\frac{\left\|\mathbf{c}_{1}-\mathbf{c}_{2}\right\|^{2}}{2 \sigma^{2}}\right) .
$$

where $c_{1}$ and $c_{2}$ are two samples and $\sigma$ is a free parameter. The polynomial kernel is expressed as:

$$
K\left(\mathbf{c}_{1}, \mathbf{c}_{2}\right)=\left(\mathbf{c}_{1}^{T} \mathbf{c}_{2}+\delta_{1}\right)^{d}
$$

where $\delta_{1}$ is a free parameter and $d$ is the order number. The sigmoid kernel is expressed as:

$$
K\left(\mathbf{c}_{1}, \mathbf{c}_{2}\right)=\tanh \left(\delta_{2} \mathbf{c}_{1}^{T} \mathbf{c}_{2}+\delta_{3}\right)
$$

where $\delta_{2}$ and $\delta_{3}$ are free parameters. It can be seen from Table 1 that the number of support vectors for different gear operating conditions is different with different kernel functions. Generally, the smaller the number of support vectors, the better the generalisation ability of the SVM and the lower the fault identification accuracy, and vice versa. Although the polynomial kernel function produced the largest number of support vectors among the three kernels, its performance on fault identification was not the best. The reason is probably that its generalisation ability is the lowest among the three kernels. Since the radial basis function had a reasonable penalty factor and support vector number, the SVM with the radial basis function achieved the best fault recognition rate of $87.75 \%$ in the present work.

From Table 2 it can be seen that, since the bispectrum of the normal gear is significantly different from the other three faulty gear conditions, the recognition rate of a normal condition is higher than that for the faulty conditions. In Figure 9, it can be observed that the bispectrum distributions of a cracked tooth and a broken tooth are similar, so the recognition rates of these two fault types are relatively low. It also can be seen in Table 2 that the fault detection performance using the radial basis function outperforms the other two kernel functions. As a result, the SVM with the radial basis function can provide satisfactory fault detection based on the

\begin{tabular}{|c|c|c|c|c|c|c|}
\hline \multirow{2}{*}{$\begin{array}{l}\text { Kernel } \\
\text { function }\end{array}$} & \multirow{2}{*}{$\begin{array}{l}\text { Penalty } \\
\text { factor }\end{array}$} & \multicolumn{4}{|c|}{ Number of support vectors } & \multirow{2}{*}{$\begin{array}{c}\text { Fault } \\
\text { detection } \\
\text { rate }(\%)\end{array}$} \\
\hline & & $\begin{array}{l}\text { Normal } \\
\text { gear }\end{array}$ & $\begin{array}{l}\text { Broken } \\
\text { tooth }\end{array}$ & $\begin{array}{l}\text { Missing } \\
\text { looth }\end{array}$ & $\begin{array}{l}\text { Cracked } \\
\text { tooth }\end{array}$ & \\
\hline Polynomial & 11.83 & 38 & 45 & 37 & 42 & 83.75 \\
\hline Radial basis & 9.82 & 21 & 15 & 23 & 28 & 87.75 \\
\hline Sigmoid & 13.78 & 11 & 22 & 29 & 19 & 82.25 \\
\hline
\end{tabular}
proposed image-based method.
Table 1. Fault detection results using the SVM with different structural parameters

\section{Conclusions}

A new image-based method has been proposed in this paper for diagnosing gear faults in fibre manufacturing lines. The innovation of the proposed method is that the wavelet packet bispectrum analysis and image fusion technique are integrated to extract distinct fault features from the bispectrum images of the gear vibration signals. To the best knowledge of the authors, the proposed image-based gear fault diagnosis method does not occur in the existing literature. Experimental data acquired from a real-world fibre manufacturing line have been used to verify the effectiveness of the proposed method. The analysis results demonstrate that: the proposed imagebased method is capable of identifying different gear faults with satisfactory detection rates; the proposed image-fusion algorithm based on the wavelet transform and matching degree can capture distinct and informative characteristics of the gear faults from the bispectrum images; and the extracted fault features based on the GLCM are effective and efficient for the SVM to detect different fault types, namely a broken tooth, missing tooth or cracked tooth. The fault detection rate using the SVM with a radial basis function 
reached $87.75 \%$. Hence, the proposed image-based method has great importance for gear fault detection in practical applications. Also, the time-frequency spectrum has good texture characteristics, so spectral pattern recognition based on time-frequency analysis technology is also of great research value. From the findings of this research, it can be seen that the extraction of features from the comprehensive description of the gear fault spectrum can improve the recognition accuracy. Future research will develop a practical system based on the proposed method for condition monitoring of gears in fibre manufacturing lines. The performance of the proposed WPBA method will also be evaluated for bearing fault diagnosis.

\section{Acknowledgements}

This project is supported by the Key Laboratory of Expressway Construction Machinery of Shaanxi Province (No 310825161123), NSFC (No 51505475), Yingcai Project of CUMT (YC2017001), Priority Academic Program Development of Jiangsu Higher Education Institutions and the UOW Vice-Chancellor's Postdoctoral Research Fellowship.

\section{References}

1. P McFadden and W Wang, 'Early detection of gear failure by vibration analysis. II. Interpretation of the time-frequency distribution using image processing techniques', Mechanical Systems and Signal Processing, Vol 7, No 3, pp 205-215, 1993.

2. A Glowacz and Z Glowacz, 'Diagnosis of the three-phase induction motor using thermal imaging', Infrared Physics \& Technology, Vol 81, pp 7-16, 2017.

3. A Glowacz, W Glowacz, Z Glowacz and J Kozik, 'Early fault diagnosis of bearing and stator faults of the single-phase induction motor using acoustic signals', Measurement, Vol 113, pp 1-9, 2018.

4. J Cheng, D Yu, J Tang and Y Yang, 'Application of frequency family separation method based on EMD and local Hilbert energy spectrum method to gear fault diagnosis', Mechanism and Machine Theory, Vol 43, No 6, pp 712-723, 2008.

5. Y Lei, Z Liu, J Ouazri and J Lin, 'A fault diagnosis method of rolling element bearings based on CEEMDAN', Proceedings of the Institution of Mechanical Engineers, Part C: Journal of Mechanical Engineering Science, Vol 231, No 10, pp 1804$1815,2015$.

6. A Glowacz and Z Glowacz, 'Diagnostics of stator faults of the single-phase induction motor using thermal images, MoASoS, and selected classifiers', Measurement, Vol 93, pp 86-93, 2016.

7. Z Li, Y Jiang, C Hu and Z Peng, 'Recent progress on decoupling diagnosis of hybrid failures in gear transmission systems using vibration sensor signal: a review', Measurement, Vol 90, pp 4-19, 2016.

8. P Hreha, A Radwanska, L Knapcikova, G Krolczyk, S Legutko, J Krolczyk, S Hloch and P Monka, 'Roughness parameters calculation by means of online vibration monitoring emerging from AWJ interaction with the material', Metrology and Measurement Systems, Vol 2, pp 315-26, 2015.
9. Z Li, X Yan, X Wang and Z Peng, 'Detection of gear cracks in a complex gearbox of wind turbines using supervised bounded component analysis of vibration signals collected from multichannel sensors', Journal of Sound and Vibration, Vol 371, pp 406-433, 2016.

10. W Wang and K Albert, 'Autoregressive model-based gear fault diagnosis', Journal of Vibration and Acoustics, Vol 124, No 4, pp 172-179, 2004.

11. G Ibrahim and A Albarbar, 'Comparison between Wigner-Ville distribution and empirical mode decomposition vibrationbased techniques for helical gearbox monitoring', Proceedings of the Institution of Mechanical Engineers, Part C: Journal of Mechanical Engineering Science, Vol 225, No 8, pp 1833-1846, 2011.

12. D Jena, S Sahoo and S Panigrahi, 'Gear fault diagnosis using active noise cancellation and adaptive wavelet transform', Measurement, Vol 47, No 5, pp 356-372, 2014.

13. P Hreha, A Radwanska, S Hloch, V Perzel, G Krolczyk and K Monkova, 'Determination of vibration frequency depending on abrasive mass flow rate during abrasive water jet cutting, The International Journal of Advanced Manufacturing Technology, Vol 77, pp 763-774, 2015

14. S C Liu and S Y Liu, 'An efficient expert system for machine fault diagnosis', International Journal of Advanced Manufacturing Technology, Vol 21, No 9, pp 691-698, 2003.

15. Y Jiang, Z Li, C Hu, C Zhang and Z Peng, 'On the bi-dimensional variational decomposition applied to non-stationary vibration signals in rolling bearing crack detection', Measurement Science and Technology, Vol 27, No 6, p 065103, 2016.

16. Y Wang and Y Cheng, 'An approach to fault diagnosis for gearbox based on image processing', Shock and Vibration, Vol 2016, p 5898052, 2016.

17. S Radhika, Y Tamura and M Matsui, 'Cyclone damage detection on building structures from pre- and post-satellite images using wavelet-based pattern recognition', Journal of Wind Engineering and Industrial Aerodynamics, Vol 136, pp 23-33, 2015.

18. S Mallat, A Wavelet Tour of Signal Processing, Second Edition, Academic Press, USA, 1998.

19. Z Li, X Yan, C Yuan, J Zhao and Z Peng, 'Fault detection and diagnosis of a gearbox in marine propulsion systems using bispectrum analysis and artificial neural networks', Journal of Marine Science and Application, Vol 10, No 1, pp 17-24, 2011.

20. L Soh and C Tsatsoulis, 'Texture analysis of SAR sea ice imagery using grey-level co-occurrence matrices', IEEE Transactions on Geoscience and Remote Sensing, Vol 37, No 2, pp 780-795, 1999.

21. A Glowacz, 'Recognition of acoustic signals of loaded synchronous motor using FFT, MSAF-5 and LSVM', Archives of Acoustics, Vol 40, No 2, pp 197-203, 2015.

22. J Wang, S Chen and C Zhang, 'Fault diagnosis method of gear based on VMD and multi-feature fusion', Mechanical Transmission, Vol 41, No 3, pp 160-165, 2015. 